\title{
ON THE POSSIBILITY OF AN INTERSUBBAND LASER IN SILICON-ON-INSULATOR
}

\author{
SERGE LURYI \\ Department of Electrical and Computer Engineering, \\ SUNY, Stony Brook, NY 11794-2350, USA \\ ALEX ZASLAVSKY \\ Division of Engineering and Physics Department, \\ Brown University, Providence, RI 02912, USA
}

\begin{abstract}
As thin Si quantum wells with oxide barriers have become an experimental reality in silicon-on-insulator technology, we discuss the feasibility of a terahertz laser based on an intersubband transition in such a Si quantum well. Electrons tunnel into an upper twodimensional subband from a thin polysilicon gate through an ultrathin tunneling oxide and are extracted laterally from the Si well by diffusion. Population inversion arises because lateral diffusion to the contacts can be a faster process than intersubband relaxation and also because the in-plane diffusivity in the upper subband is suppressed by the interaction with a nearby subband characterized by a heavy in-plane mass. Thick oxide layers provide optical confinement and the main optical loss mechanism is the weak free-carrier absorption in thin doped regions in the polysilicon gate and substrate. A voltage on the substrate may provide some tunability of the gain peak.
\end{abstract}

\section{Introduction}

Optical sources based on intersubband transitions in bandgap-engineered semiconductor quantum wells $(\mathrm{QW})$, originally proposed by Kazarinov and Suris, ${ }^{1}$ are undergoing spectacular development, with quantum cascade lasers (QCLs) improving in power and temperature performance, ${ }^{2,3}$ as well as reaching into the long-wavelength $\mathrm{THz}$ regime. ${ }^{4,5,6}$ All of the existing QCL designs are based on epitaxially grown III-V semiconductor heterostructures systems, with aggressive bandgap engineering requiring monolayer control over thickness and composition. In a typical QCL design, the upper level of the intersubband transition $E_{2}$ is filled by tunneling from an adjacent injector region, while the lower level $E_{1}$ is emptied out by tunneling into an adjacent state or miniband downstream. The active structure is repeated 20-100 times, separated by doped carrier 
reservoirs that ensure the overall charge neutrality under bias and prevent the formation of high electric field domains. ${ }^{7}$ The main physical issues faced by QCL lasers include the competing non-radiative $E_{2} \rightarrow E_{1}$ transitions that greatly increase the current density required for population inversion, difficulties in rapidly removing carriers from the $E_{1}$ subband, and optical losses due to absorption within the QCL structure. Another requirement is the optical confinement in the vertical direction outside the structure. ${ }^{8}$

Unlike interband semiconductor lasers that require a direct bandgap, intersubband optical sources are, in principle, compatible with dominant Si technology. However, the most advanced Si-based heterostructure, $\mathrm{Si} / \mathrm{SiGe}$, is hampered by relatively low barriers, lattice mismatch strain, and easier bandgap engineering in the valence band where the effective masses are heavy. As a result, while there has been encouraging progress on electroluminescence from $\mathrm{Si} / \mathrm{SiGe} \mathrm{QW}$ structures, ${ }^{9,10}$ their optical efficiency has lagged behind III-V designs and the insertion of such a structure into an optical waveguide has not been reported to date. In this letter we propose an alternative Si-compatible approach based on the silicon-on-insulator (SOI) technology that is predicted to become dominant in mainstream digital circuitry in the near future. ${ }^{11}$

\section{Proposed Device Structure and Operation}

The crucial ingredients, now available in SOI technology, are an ultrathin Si QW channel and $\mathrm{SiO}_{2}$ tunneling gate oxide, combined with lateral current extraction from the QW. The proposed device structure is schematically illustrated in Fig. 1: it is essentially a standard SOI transistor with gate length $L_{\mathrm{G}}=2 L$, but with a Si channel thinned to $6 \mathrm{~nm}$, an ultrathin tunneling gate oxide of $\sim 1 \mathrm{~nm}$, a thin in-situ doped poly-Si gate, and a deposited oxide layer on top. Outside the gate region, there are thicker contact regions (in a standard transistor these would be the source and drain). The electronic properties of a similar vertical tunneling transistor (VTT) structure, fabricated by a fully technological SOI process, were recently reported. ${ }^{12}$ In order to minimize losses due to free-carrier absorption discussed later, the poly-Si gate is only $20 \mathrm{~nm}$ thick and relatively lightly doped $3 \times 10^{17} \mathrm{~cm}^{-3}$; whereas the Si substrate is minimally doped, with a thin $\sim 20$ $\mathrm{nm}$ doped layer under the buried oxide to which a back-gate bias $V_{\mathrm{BG}}$ can be applied.

The band diagram of the device along a vertical cut, as well as the principle of operation, are illustrated in Fig. 2, with the corresponding electronic subband structure $E_{\mathrm{N}}$ in the quantum well shown in the inset. Electrons tunnel from the gate electrode into the quantized 2D subbands in the $\mathrm{Si} \mathrm{QW}$ and are extracted laterally via the contacts. As in all resonant tunneling structures, the elastic tunneling into the QW conserves energy 


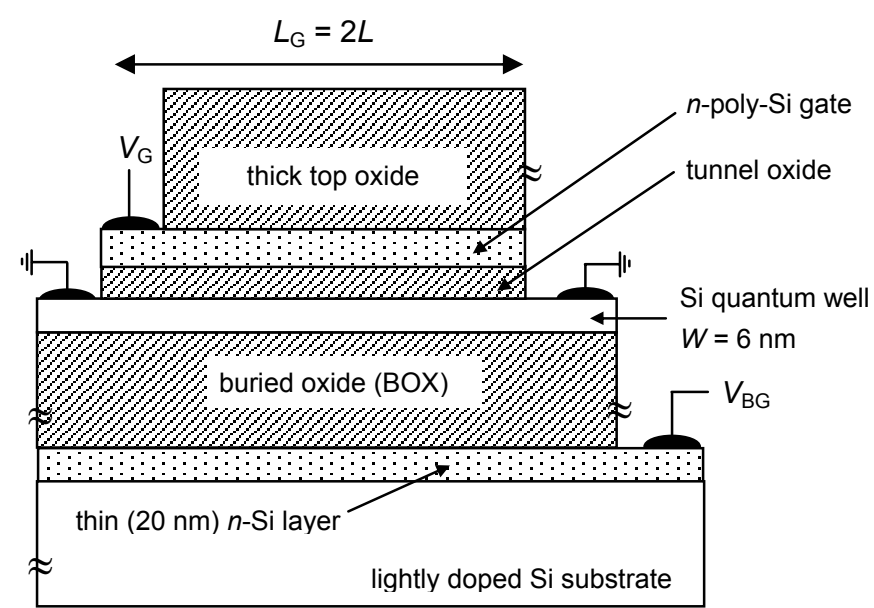

Fig. 1. Cross-sectional view of the SOI device (not to scale): $n$-poly-Si gate is doped $3 \times 10^{17} \mathrm{~cm}^{-3}$ and is $20 \mathrm{~nm}$ thick to reduce free-carrier absorption; undoped Si quantum well $W=6 \mathrm{~nm}$; tunneling oxide is $\sim 1 \mathrm{~nm}$; thick $\mathrm{SiO}_{2}$ layers sandwich the active structure; there is a thin $20 \mathrm{~nm} n^{+}$-Si layer under the buried oxide, with the rest of the $\mathrm{Si}$ substrate taken as insulating at $T=77 \mathrm{~K}$.

and transverse momentum, ${ }^{13}$ and depends on the alignment of $E_{\mathrm{N}}$ with the occupied states in the gate. This alignment can be tuned slightly by a voltage on the back-gate electrode $V_{\mathrm{BG}}$ due to the quantum capacitance effect, ${ }^{14}$ as was first demonstrated in III-V ${ }^{15}$ and, recently, $\mathrm{SOI}^{12}$ device structures. The total thickness of the active structure, including the poly-Si gate, very thin oxide tunnel barrier, and Si QW, is $d=25 \mathrm{~nm}$.

Now consider what happens when the electrons are injected into the higher-lying $E_{2}$ subband of the Si QW corresponding to the heavy effective mass $m_{1}^{*} \sim 0.98 m_{0}$ in $z$ direction (where $m_{0}$ is the free electron mass). The electrons will diffuse laterally in the $E_{2}$ subband towards the contacts, a process characterized by some diffusion coefficient $D_{2}$. They will relax to the lowest $E_{1}$ subband with a characteristic lifetime $\tau$ and these carriers will also diffuse towards the contacts, with a different diffusion coefficient $D_{1}$. As long as the energy separation $\left(E_{2}-E_{1}\right)$ lies below the Si optical phonon energy, nonradiative relaxation to $E_{1}$ is suppressed. Let the injection current density into the second subband be $J(x)$. The equations describing carrier transport in the two subbands are given by current continuity: 


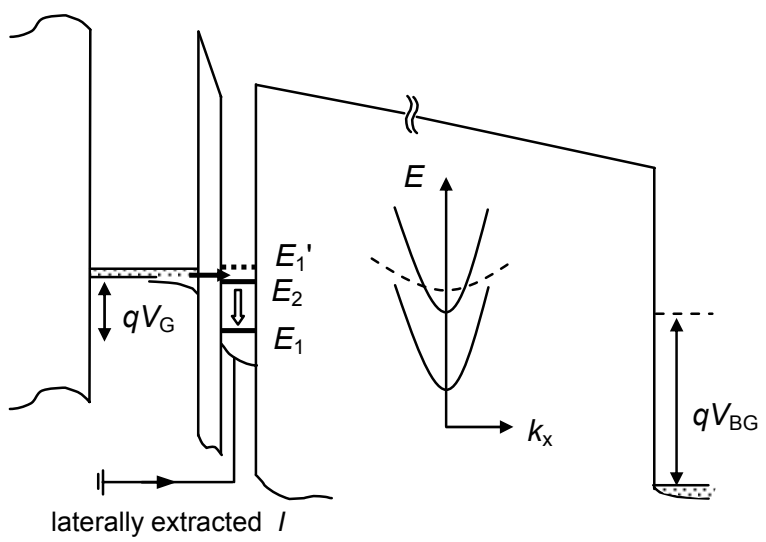

Fig. 2. Schematic band diagram of the device under bias. The Si quantum well provides an energy separation $\hbar \omega=\left(E_{2}-E_{1}\right)<\hbar \omega_{\mathrm{LO}}$ between the lowest doubly-degenerate electron 2D subbands. Electrons tunnel into $E_{2}$ from the gate and diffuse laterally to the source and drain contacts. More rapid diffusion in the $E_{1}$ subband contributes to the population inversion. The alignment of $E_{2}$ with the occupied states in the gate can be tuned by the back-gate bias $V_{\mathrm{BG}}$, but the thick buried oxide prevents current leakage to the substrate. Inset shows the schematic in-plane dispersion $E\left(k_{\mathrm{x}}\right)$ of the confined 2D subbands in the Si QW. The fourfold-degenerate subband $E_{1}{ }^{\prime}$ corresponding to the heavier transverse $\mathrm{Si}$ mass lies above $E_{2}$. Coupling between $E_{2}$ and $E_{1}{ }^{\prime}$ reduces the in-plane diffusivity in the upper level. Optical transitions are only possible between $E_{2}$ and $E_{1}$, as $E_{1}{ }^{\prime}$ lies in a different valley in $k$-space.

$$
\begin{aligned}
& \partial I_{1} / \partial x=-D_{1} \partial^{2} n_{1} / \partial x^{2}=n_{2}(x) / \tau, \text { and } \\
& \partial I_{2} / \partial x=-D_{2} \partial^{2} n_{2} / \partial x^{2}=J(x)-n_{2}(x) / \tau,
\end{aligned}
$$

where $I_{1}$ and $I_{2}$ are the channel currents flowing in the first and second subbands, whereas $n_{1}(x)$ and $n_{2}(x)$ are the corresponding carrier sheet densities. By symmetry of the device in Fig. 1, we need only consider half of the structure, $0 \leq x \leq L$. The second order system of differential equations (1) and (2) obeys the following boundary conditions: $n_{1}(x)=n_{2}(x)=0$ at $x=L$ and $\partial n_{1} /\left.\partial x\right|_{x=0}=\partial n_{2} /\left.\partial x\right|_{x=0}=0$. The first of these boundary conditions assumes a perfect carrier sink in the contacts, while the second reflects the symmetry of the device around the $x=0$ midpoint.

Adding equations (1) and (2) and integrating, using the boundary conditions describe above, and assuming $J(x)$ to be a constant $J_{0}$ we obtain: 


$$
D_{1} n_{1}(x)+D_{2} n_{2}(x)=\frac{J_{0} L^{2}}{2}\left(1-\frac{x^{2}}{L^{2}}\right)
$$

The assumption of a constant $J(x)=J_{0}$ is reasonable as long as the local potential in the channel does not change along $x$, but an expression similar to Eq. (3) involving an integral of $J(x)$ over the channel length will hold for any $J(x)$. Now we turn to Eq. (2). In the absence of injection, the homogeneous equation has the usual diffusion solution $\exp \left( \pm x / L_{\mathrm{D}}\right)$, where $L_{\mathrm{D}} \equiv\left(D_{2} \tau\right)^{1 / 2}$ is the diffusion length with respect to relaxation to $E_{1}$. The inhomogeneous solution is $n_{2}=-J_{0} \tau$ and, imposing the boundary conditions, we obtain the following expressions:

$$
\begin{aligned}
& n_{2}(x)=J_{0} \tau\left(1-\frac{\cosh \left(x / L_{\mathrm{D}}\right)}{\cosh \left(L / L_{\mathrm{D}}\right)}\right), \text { and } \\
& n_{1}(x)=\frac{J_{0} L^{2}}{2 D_{1}}\left(1-\frac{x^{2}}{L^{2}}\right)-\frac{D_{2}}{D_{1}} n_{2}(x) .
\end{aligned}
$$

For $L_{\mathrm{D}}>L$, we can expand the hyperbolic cosines in Eq. (4) up to $\left(L / L_{\mathrm{D}}\right)^{4}$ to obtain the following estimate for the population inversion:

$$
\frac{n_{2}(x)}{n_{1}(x)}=\frac{D_{1}}{D_{2}}\left(\frac{12 L_{\mathrm{D}}^{2}}{5 L^{2}-x^{2}}-1\right) .
$$

Note that in the $L_{\mathrm{D}}>L$ limit, Eq. (6) predicts population inversion even for equal diffusion coefficients $D_{1}=D_{2}$, because the electrons do not have time to relax from $E_{2}$ to $E_{1}$ during their diffusion towards the contacts. The ratio $D_{1} / D_{2}$ increases the inversion further, as the in-plane diffusivity $D_{2}$ is expected to be markedly smaller because of the interaction with the nearby $E_{1}$ ' subband (corresponding to the light effective mass in the $z$ direction - see Fig. 2) that has a heavy in-plane mass and a large density of states. Given that optical phonon scattering is turned off in our structure, as $\left(E_{2}-E_{1}\right)<\hbar \omega_{\mathrm{LO}}$, the main nonradiative relaxation processes are acoustic-phonon assisted or the weaker multielectron Auger-like processes. In a similarly narrow GaAs QW, the acoustic phonon intersubband scattering time at $77 \mathrm{~K}$ was calculated to be in the several tens of ps range, implying $L_{\mathrm{D}}$ of several $\mu \mathrm{m} .^{16}$

Next we consider the other necessary constituents of an effective infrared source, using a typical vertical III-V QCL design for comparison. Given the intersubband nature of the $E_{2} \rightarrow E_{1}$ transition, the optical matrix element in our Si QW is, in principle, quite similar to that in a GaAs QW. In order to avoid competing nonradiative relaxation via 
phonon emission, the Si QW in Fig. 2(b) should not be too narrow, e.g. in a $6 \mathrm{~nm} \mathrm{QW}$, $\left(E_{2}-E_{1}\right) \sim 30 \mathrm{meV}<\hbar \omega_{\mathrm{LO}}$ in Si. In a vertical QCL design, the upper level can be emptied by tunneling into available states downstream - a problem that has often been addressed by bandgap engineering a minigap aligned with the upper lasing state. ${ }^{2}$ In our proposed device, the second barrier is completely impenetrable, see Fig. 2, and measurements on the similar VTT design have shown no measurable substrate leakage current for any $V_{\mathrm{BG}}{ }^{12}$ Optical mode confinement for far infrared wavelengths has been a major issue in III-V QCL designs because of the relatively small change in refractive index available in the epitaxial III-V heterostructures. Either the combination of a metallic contact on top and a heavily-doped surface-plasmon-supporting semiconductor contact layer on the bottom ${ }^{4,5}$ or even all-metal confinement (involving the bonding of the active QCL structure to a metallized substrate, followed by subsequent processing) ${ }^{6}$ has been employed. At the same time, the active region in QCL is repeated many times to bring the total active thickness closer to $\lambda$, increasing the gain to counter the additional optical losses due to incomplete confinement. In our device, the structure is based on a single QW and cannot easily be repeated, due to the SOI substrate fabrication technology. As a result, the optical confinement $\Gamma$ at $\lambda=40 \mu \mathrm{m}$ is very weak, despite the large refractive index discontinuity between $\mathrm{Si}$ and $\mathrm{SiO}_{2}:{ }^{17}$

$$
\Gamma \sim 2 \pi^{2}\left(\varepsilon_{\mathrm{SiO} 2} / \varepsilon_{\mathrm{Si}}\right)\left(\varepsilon_{\mathrm{Si}}-\varepsilon_{\mathrm{SiO} 2}\right)(d / \lambda)^{2} \sim 10^{-3}
$$

where $d=25 \mathrm{~nm}$. Consequently, there is no significant difference between the optical field intensity in the Si QW gain region and the doped poly-Si gate and substrate regions, which are the regions of optical loss due to free-carrier absorption.

The gain in the Si quantum well can be estimated from the usual intersubband absorption expression: ${ }^{18}$

$$
\alpha(\omega)=\frac{8 \pi^{2} e^{2}\left(E_{2}-E_{1}\right)}{n_{\mathrm{Si}} h c \Delta E}\left(n_{2}-n_{1}\right) \frac{\left|z_{12}\right|^{2}}{W}
$$

where $\Delta E$ is the subband energy broadening, $n_{\mathrm{Si}}$ is the refractive index of $\mathrm{Si}, c$ is the speed of light, and $\left|z_{12}\right|$ is the overlap integral $\left\langle\chi_{2}|z| \chi_{1}\right\rangle$ (in an infinite quantum well of width $\left.W,\left|z_{12}\right| / W=16 / 9 \pi^{2}\right)$. Estimating the energy broadening in the Si QW to be $\Delta E \sim 5$ $\mathrm{meV}$, corresponding to monolayer fluctuations in the well width $W$, and assuming strong population inversion $\left(n_{2}-n_{1}\right) \approx n_{2}$ as predicted by Eq. (6), we obtain a gain $\alpha \sim$ $350\left(n_{2} / 10^{11}\right) \mathrm{cm}^{-1}$ for our $W=5 \mathrm{~nm}$ Si quantum well.

The optical losses in our device are due to free-carrier absorption in the poly-Si 
emitter, narrow doped region under the buried oxide (BOX), and the lateral contacts, as well as possible absorption in the $\mathrm{SiO}_{2}$ cladding layers. Free-carrier absorption in heavily doped semiconductors $\alpha_{\mathrm{L}} \sim N \lambda^{2}$, where $N$ is the carrier concentration. ${ }^{19}$ In $n$-Si, absorption in the infrared was measured by Spitzer and Fan: ${ }^{20}$ at $T=77 \mathrm{~K}$ the absorption for Si doped $N_{\mathrm{D}} \sim 3 \times 10^{17} \mathrm{~cm}^{-3}$ is roughly $\alpha_{\mathrm{L}} \sim 150 \mathrm{~cm}^{-1}$ when extrapolated to $\lambda=40 \mu \mathrm{m}$. Since the total thickness of the doped regions in our device is $40 \mathrm{~nm}$ whereas the quantum well $W=6 \mathrm{~nm}$, to overcome the free-carrier absorption losses we need to get the sheet density $n_{2}$ in the upper $E_{2}$ subband to reach at least $3 \times 10^{11} \mathrm{~cm}^{-2}$. The tunneling current densities measured in the similarly designed VTT structure ${ }^{12}$ were far too small to achieve such a charge density in the well, but a thinner $\mathrm{SiO}_{2}$ tunnel barrier or possibly an alternative dielectric barrier with a smaller barrier height ${ }^{21}$ could be used to increase the current density. As for infrared absorption in $\mathrm{SiO}_{2}$, the lowest-energy absorbing mode in the IR appears to be the Si-O rocking vibration at $\sim 400 \mathrm{~cm}^{-1}$, leading to strong absorption near $\lambda=25 \mu \mathrm{m}$ that dies out exponentially at longer wavelengths. ${ }^{22}$ As a result, operation at $\lambda=40 \mu \mathrm{m}$ should still be feasible, although it is interesting to note that $\mathrm{SiO}_{2}$ absorption poses a more stringent requirement on the minimum $\lambda$ of our proposed device than the LO phonon energy in the Si QW.

The remaining question regarding the feasibility of SOI-based unipolar laser concerns the uniformity of the Si QW on top of the BOX layer. The possibility of thinning the $\mathrm{Si}$ channel by means of repeated sacrificial oxidation is a relatively recent development, ${ }^{23,24}$ and the near-perfect control of layer thickness by epitaxy, crucial to existing III-V QCL designs, is not yet attainable. As a result, devices based on the existence of sharp 2D quantization in the QW suffer from the subband broadening due to QW thickness nonuniformity. ${ }^{12}$ In our device, given a reasonable carrier density in the well, the lateral diffusion of these electrons to the contact regions will not be affected by the local potential dips and hills due to thickness fluctuations. At the same time, recent reports of photoluminescence from $\mathrm{Si}$ QW down to $2 \mathrm{~nm}$ thickness sandwiched between $\mathrm{SiO}_{2}$ barriers on SOI indicate that thickness nonuniformity is dropping towards a single monolayer. ${ }^{25}$ It is this monolayer nonuniformity that we assumed in Eq. (6) to estimate the available gain.

\section{Conclusion}

We have proposed an intersubband laser in SOI, based on tunneling of electrons into a higher-lying quantized subband of a Si QW followed by their lateral diffusion and extraction at the contacts. The slow nonradiative relaxation between $\mathrm{QW}$ subbands that are separated by less than the optical phonon energy leads to a reasonable diffusion 
length $L_{\mathrm{D}}$ and significant population inversion. The diffusivity in the upper level of the intersubband transition is smaller than in the lower level, further contributing to the population inversion. Despite weak optical confinement at $\lambda \sim 40 \mu \mathrm{m}$, for reasonable QW carrier density the gain is sufficient to overcome the free-carrier absorption losses in the thin doped regions of the device, e.g. the poly-Si gate layer from which the electrons tunnel into the QW. The necessary technological ingredients for our device, such as a uniform ultrathin Si QW on SOI, are almost within reach of modern SOI technology.

\section{Acknowledgements}

The work at Brown and SUNY-Stony Brook has been supported by the AFOSR MURI program (award \# F49620-00-1-0331, managed by T. Steiner). We acknowledge helpful discussions with Prof. R. Beresford and Dr. R. Soref.

\section{References}

1 R. F. Kazarinov and R. Suris, "Electric and electromagnetic properties of semiconductors with a superlattice", Sov. Phys. Semicond. 5, 797 (1971).

2 J. Faist, F. Capasso, C. Sirtori, D. L. Sivco, A. L. Hutchinson, and A. Y. Cho, "Quantum cascade laser", Science 264, 553 (1994)

3 J. S. Yu, S. Slivken, A. Evans, L. Doris, and M. Razeghi, "High-power c-w operation of a 6 $\mu \mathrm{m}$ quantum cascade laser at room temperature", Appl. Phys. Lett. 83, 2503 (2003).

4 R. Köhler, A. Tredicucci, F. Beltram, H. Beere, E. Linfield, A. Davies, D. Ritchie, R. Iotti, and F. Rossi, "Terahertz semiconductor-heterostructure laser", Nature 417, 156 (2002).

5 M. Rochat, L. Ajili, H. Willenberg, J. Faist, H. Beere, G. Davies, E. Linfield, and D. Ritchie, "Low-threshold terahertz quantum-cascade lasers", Appl. Phys. Lett. 81, 1381 (2002).

6 B. Williams, H. Callebaut, S. Kumar, Q. Hu, and J. Reno, "3.4 THz quantum cascade laser based on longitudinal-optical-phonon scattering for depopulation", Appl. Phys. Lett. 82, 1015 (2003).

7 A. Kastalsky, V. J. Goldman, and J. H. Abeles, "Possibility of infrared laser in resonanttunneling structure", Appl. Phys. Lett. 59, 2636 (1991).

8 An up-to-date discussion of THz cascade lasers is available in chapter 3 of Future Trends in Microelectronics: The Nano, the Giga, and the Ultra, S. Luryi, J. M. Xu, and A. Zaslavsky, eds., (Wiley, New York, 2004), pp. 283-390. 

E. Müller, "Intersubband photoluminescence from silicon-based quantum cascade structures", Science 290, 2277 (2000).

10 L. Diehl, S. Mentese, E. Müller, D. Grützmacher, H. Sigg, U. Gennser, I. Sagnes, Y. Campidelli, O. Kermarrec, D. Bensahel, and J. Faist, "Electroluminescence from straincompensated $\mathrm{Si}_{0.2} \mathrm{Ge}_{0.8} / \mathrm{Si}$ quantum-cascade structures based on a bound-to-continuum transition", Appl. Phys. Lett. 81, 4700 (2002).

11 For an overview of SOI technology, see G. K. Celler and S. Cristoloveanu, "Frontiers of silicon-on-insulator", J. Appl. Phys. 93, 4955 (2003).

12 A. Zaslavsky, C. Aydin, S. Luryi, S. Cristoloveanu, D. Mariolle, D. Fraboulet, and S. Deleonibus, "Ultrathin silicon-on-insulator vertical tunneling transistor", Appl. Phys. Lett. 83, 1653 (2003).

13 S. Luryi, "Frequency limit of double-barrier resonant-tunneling oscillators", Appl. Phys. Lett. 47, 490 (1985).

14 S. Luryi, "Quantum capacitance devices", Appl. Phys. Lett. 52, 501 (1988).

15 F. Beltram, F. Capasso, S. Luryi, S.-N. G. Chu, A. Y. Cho, and D. L. Sivco, "Negative transconductance via gating of the quantum-well subbands in a resonant tunneling transistor", Appl. Phys. Lett. 53, 219 (1988).

16 J. H. Smet, C. G. Fonstad, and Q. Hu, "Intrawell and interwell intersubband transitions in multiple quantum wells for far-infrared sources", J. Appl. Phys. 79, 9305 (1996).

17 H. C. Casey, Jr. and M. B. Panish, Heterostructure Lasers. Part A: Fundamental Principles (Academic Press, New York, 1978), pp. 54-57.

18 A. Ya. Shik, "Optical properties of semiconductor superlattices with complex band structures", Sov. Phys. Semicond. 6, 1110 (1973).

19 K. Seeger, Semiconductor Physics (Wiley, New York, 1973).

20 W. Spitzer and H. Y. Fan, "Infrared absorption in $n$-type silicon", Phys. Rev. 108, 268 (1957). The samples measured in that study were quite compensated, with carrier density $n<N_{\mathrm{D}}$. Since free-carrier absorption is inversely proportional to mobility, a modern clean $\mathrm{Si}$ sample would exhibit less absorption for the same free-carrier density.

21 S. Luryi and A. Zaslavsky, "Blue sky in SOI: new opportunities for quantum and hot-electron devices", Solid State Electronics 48, 877 (2004) discusses the use of low-barrier height dielectrics for tunneling barriers in SOI quantum devices.

22 D. Tsu, "Infrared optical constants of silicon dioxide thin films by measurements of $R$ and $T$ ", J. Vac. Sci. Technol. B 18, 1796 (2000); H. R. Philipp, "The infrared optical properties of $\mathrm{SiO}_{2}$ 
and $\mathrm{SiO}_{2}$ layers on silicon", J. Appl. Phys. 50, 1053 (1979). We are grateful to Dr. Richard Soref for drawing our attention to the possibility of infrared absorption in $\mathrm{SiO}_{2}$.

23 E. Suzuki, K. Ishii, S. Kanemaru, T. Maeda, T. Tsutsumi, T. Sekigawa, K. Nagai, and H. Hiroshima, "Highly suppressed short-channel effects in ultrathin SOI $n$-MOSFETs", IEEE Trans. Electron Dev. 47, 354 (2000).

24 D. Esseni, M. Mastrapasqua, G. K. Celler, F. H. Baumann, C. Fiegna, L. Selmi, and E. Sangiorgi, "Low field mobility of ultrathin SOI $n$ - and $p$-MOSFETs: Measurements and implications on the performance of ultra-short MOSFETs," Tech. Digest IEDM (2000).

25 M. A. Green, "Silicon-on-insulator (SOI): A path for integration of silicon light emitters into future microelectronic chips?", in: Future Trends in Microelectronics: The Nano, the Giga, and the Ultra, S. Luryi, J. M. Xu, and A. Zaslavsky, eds., (Wiley, New York, 2004), pp. 243252. 